\title{
Understanding the physical examination of the shoulder: a narrative review
}

\author{
Seoyon Yang ${ }^{1}$, Tae Uk Kim², Du Hwan Kim ${ }^{3}$, Min Cheol Chang ${ }^{4}$ \\ ${ }^{1}$ Department of Rehabilitation Medicine, Ewha Woman's University Seoul Hospital, Ewha Woman's University School of Medicine, Seoul, Korea; \\ ${ }^{2}$ Department of Rehabilitation Medicine, Dankook University College of Medicine, Cheonan, Republic of Korea; ${ }^{3}$ Department of Physical Medicine \\ and Rehabilitation, College of Medicine, Chung-Ang University, Seoul, Korea; ${ }^{4}$ Department of Rehabilitation Medicine, College of Medicine, \\ Yeungnam University, Daegu, Republic of Korea \\ Contributions: (I) Conception and design: All authors; (II) Administrative support: None; (III) Provision of study materials or patients: All authors; \\ (IV) Collection and assembly of data: All authors; (V) Data analysis and interpretation: All authors; (VI) Manuscript writing: All authors; (VII) Final \\ approval of manuscript: All authors. \\ Correspondence to: Min Cheol Chang, MD. Department of Physical Medicine and Rehabilitation, College of Medicine, Yeungnam University \\ 317-1, Daemyungdong, Namku, Taegu, 705-717, Republic of Korea. Email: wheel633@ynu.ac.kr. Du Hwan Kim, MD. Department of Physical \\ Medicine and Rehabilitation, College of Medicine, Chung-Ang University, 102, Heukseok-ro, Dongjak-gu, Seoul, 06973, Republic of Korea. \\ Email: ri-pheonix@hanmail.net.
}

\begin{abstract}
The shoulder joint is a ball and socket joint which provides an extensive range of motion. Shoulder pain and weakness are common complaints among patients, which can lead to disability and affect a person's ability to perform daily activities. Shoulder pain and weakness may be associated with shoulder conditions such as rotator cuff disorders adhesive capsulitis, superior labrum anterior to posterior lesions, lesions in the biceps, acromioclavicular joint disease, or instability. Often, a thorough understanding of the network of bony, ligamentous, muscular, and neurovascular anatomy is required to properly identify and diagnose shoulder pathology. Identifying a specific shoulder pathology may be challenging, considering the numerous structures involved in shoulder function. Appropriate physical examination of the shoulder is important for making an accurate diagnosis and distinguishing certain pathologies of the shoulder. Evaluation of shoulder problem may be reliant upon physical examination, which involves inspection, palpation, assessment of range of motion, strength, and neurovascular integrity. In addition, specific tests are used to reproduce symptoms and signs that would help physicians identify the pathology of the shoulder problem. The aim of this study was to review the shoulder anatomy and describe the specific tests used to evaluate common shoulder conditions to facilitate accurate diagnosis and guide proper treatment of these conditions.
\end{abstract}

Keywords: Shoulder; shoulder pain; physical examination; diagnosis

Submitted Sep 09, 2020. Accepted for publication Dec 08, 2020.

doi: 10.21037/apm-20-1808

View this article at: http://dx.doi.org/10.21037/apm-20-1808

\section{Introduction}

Shoulder pain and weakness, which can result in limitations in performing activities of daily living, are among the most common musculoskeletal complaints at primary care centers, with reported prevalence rates ranging from $7 \%$ to $26 \%(1,2)$. Rotator cuff disorders adhesive capsulitis, superior labrum anterior to posterior lesions, lesions in the biceps, acromioclavicular (AC) joint disease, and instability are some common shoulder conditions. Identifying a specific shoulder pathology may be challenging, considering the numerous structures involved in shoulder function. Specific tests are used to reproduce symptoms and signs that would help physicians identify the pathology of the 
shoulder problem. This article aims to review shoulder anatomy and describe special tests used to evaluate common shoulder conditions in order to facilitate accurate diagnosis and guide proper treatment of these conditions.

We present the following article in accordance with the Narrative Review reporting checklist (available at http:// dx.doi.org/10.21037/apm-20-1808).

\section{Anatomy}

To better understand shoulder pain and distinguish between the normal and pathologic conditions of the shoulder, physicians must first understand normal shoulder anatomy and function (1). This understanding can aid physicians when examining structures around the shoulder joint.

The shoulder consists of three bones and four joints. The shoulder girdle consists of the clavicle, scapular, and humeral head. Among the joints of the shoulder, which are the glenohumeral, sternoclavicular, acromioclavicular, and scapulothoracic joints, the glenohumeral joint is the major shoulder joint that involves a complex articulation between the humeral head and the glenoid cavity of the shoulder girdle. It is a ball-and-socket joint, where only $25 \%$ of the humeral head articulates with the glenoid fossa, forming an extremely shallow articulation (3). This makes the shoulder the most mobile joint in the body, and also, unstable. The rim of the glenoid is covered with the labrum, a fibrocartilage ring, which helps improve stability. Histologically, it consists of dense fibrous tissue. The labrum in the superior half of the glenoid is typically triangular in shape. About half of the biceps tendon fibers attach to the superior glenoid tubercle while the other half attach to varying degrees to the labrum, anterior and posterior to the superior glenoid tubercle (4).

The muscles around the shoulder include the deltoid, pectoralis major and minor, biceps brachii, triceps brachii, teres major, latissimus dorsi, and rotator cuff, which attach to the scapula, humerus, and clavicle. They provide a wide range of motion (ROM) and protect the glenohumeral joint. Different forces with different intensities and directions over the humeral head are delivered by the deltoid, biceps brachii, and rotator cuff muscles (5). The rotator cuff consists of four muscles; the supraspinatus, infraspinatus, subscapularis, and teres minor. Individually, the supraspinatus (abduction), subscapularis (internal rotation), infraspinatus, and teres minor (external rotation) are involved in a variety of shoulder movement. Their main role is to stabilize the shoulder and shoulder complex. The supraspinatus and subscapularis tendons are susceptible to impingement by various structures around them, such as the acromion, coracoacromial ligaments, acromioclavicular joint, or coracoid process. Subacromial impingement refers to an encroachment on the subacromial tissues due to narrowing of the subacromial space, which includes structures such as the supraspinatus tendon, the subacromial bursa, the long head of the biceps brachii tendon, and the joint capsule (6). Sustained impingement can trigger inflammatory changes that may progress to tendinosis and degeneration of these tendons, which may further proceed to tendon weakness and tear (7). Tears in the articular side of the rotator cuff are commonly associated with intrinsic degenerative changes, and those in the bursal side are commonly caused by subacromial impingement with a mildly degenerated rotator cuff (8). In other words, subacromial impingement syndrome is a continuum ranging from the inflammation of the subacromial bursa (subacromial bursitis) to rotator cuff tendinopathy and tears (9).

The nerves of the brachial plexus and subclavian vessels travel along the anterior courses along the anterior shoulder girdle between the first rib and clavicle. The brachial plexus originates from the nerve roots $\mathrm{C} 5-\mathrm{T} 1$ and divides into trunks, divisions, cords, and branches as it courses anteriorly and laterally toward the distal arm (1). It is usually found below the clavicle. The suprascapular nerve emerges from the upper trunk of the brachial plexus and travels along the superior scapula through the suprascapular notch and provides motor branches to the supraspinatus muscle. Then, it travels along the spinoglenoid notch and into the infraspinous fossa, where it supplies innervation to the infraspinatus muscle (10) A suprascapular nerve block was found to be useful in reducing postoperative pain in patients who underwent shoulder surgery (11). Compression of the nerve, for example, due to a paralabral cyst, can occasionally occur at the spinoglenoid notch, which triggers denervation of the infraspinatus muscle, resulting in its atrophy. The subscapular nerves branch out from the posterior cord to innervate the subscapularis muscle, as the axillary nerve travels to the posterior side of the shoulder through the quadrangular space. The quadrilateral space is bounded superiorly by the teres minor muscle, inferiorly by the teres major muscle, medially by the long head of the triceps, and laterally by the humeral shaft (12). The axillary and posterior humeral circumflex artery runs through the quadrilateral space. Compression of these structures by fibrous bands, trauma, or hypertrophy of a muscular border leads to quadrilateral space syndrome. This syndrome is 
characterized by the weakness or tenderness of the deltoid and teres minor muscles, pain over the anterior aspect of the shoulder, and paresthesia of the upper extremity $(13,14)$. Isolated teres minor atrophy or deltoid atrophy has been reported to be a relatively common shoulder pathology with distinct features from quadrilateral space syndrome (15). The axillary nerve is susceptible to injury in open-shoulder procedures (1). The musculocutaneous nerve branches out from the lateral cord and innervates the biceps brachii and brachialis muscles. The shoulder can be injured in the deltopectoral approach during open-shoulder surgery or manipulation of the conjoint tendon (1).

The movement of the shoulder relies on various structures around the shoulder joint, such as the bones, ligaments, tendons, muscles, and nerves. A detailed understanding of the anatomy of the shoulder is necessary to address the etiology of shoulder problems.

\section{Physical examination}

The physical examination of the shoulder begins with history taking, inspection, palpation, assessment of ROM, muscle strength tests, and physical examination tests to obtain important diagnostic clues. First, it is important to take a detailed history, which can guide further necessary exams to identify the pathology of any shoulder problem. History taking is important to rule out extrinsic etiology of shoulder pain, which is usually represented by pain that is difficult to localize and not affected by passive and active ROM. A history of trauma is likely to be related with fractures or dislocations. Typical questions to assess the characteristics of shoulder pain should include the duration, quality, associated symptoms, radiation, and aggravating and alleviating factors. It is also important to be aware of the patient's age when assessing the shoulder problem; traumatic injuries are frequently observed in young adults whereas rotator cuff diseases and adhesive capsulitis are common in the elderly (16).

The next important step is inspection, which involves looking at the entire shoulder with proper exposure. The patient should be inspected both anteriorly and posteriorly (7). The examiner should look for asymmetry between the affected and unaffected sides of the shoulder. Presence of atrophy, deformity, ecchymosis, swelling, wounds, scarring, and redness of the skin should be noted (16). In cases of massive rotator cuff tears, the humeral head can be seen as a bulge anteriorly within the shoulder (7). Any abnormalities seen on the affected side should be compared with the contralateral side.
Palpation is another important step. Finding areas of focal tenderness is important; for instance, tenderness in certain areas may represent acromioclavicular osteoarthritis, bicipital tendinitis, or rotator cuff disorders (17). Pain induced by deep palpation of the lateral deltoid inferior to the acromial process may reflect the presence of supraspinatus tendinitis or rotator cuff tear (14). Palpation of both shoulders is usually recommended, considering that certain structures of the shoulder can induce pain on palpation in the affected shoulder. Specific positions improve the reliability of palpation. For example, the modified Crass position is recommended for evaluation of the supraspinatus tendon (18). In this position, the dorsal aspect of the ipsilateral hand is placed behind the buttock, with the arm hyperextended and internally rotated. This pulls the supraspinatus tendon out from the acromion and allows improved visualization. The greater tuberosity is located slightly anteriorly, which is easy to localize (19). The supraspinatus tendon is easily visualized by ultrasonography in this position, which allows accurate identification of a tear.

Assessment of shoulder ROM should include both active (unaided) and passive (with assistance from the examiner) movements. Loss of both may indicate adhesive capsulitis, whereas loss of only active ROM reflects shoulder impingement. The ROM includes forward flexion from $150^{\circ}$ to $180^{\circ}$, extension from $40^{\circ}$ to $60^{\circ}$, abduction from $150^{\circ}$ to $180^{\circ}$, and external rotation from $60^{\circ}$ to $90^{\circ}$. Internal rotation is assessed by the vertebral level that hand can reach. The vertebral level of superior scapular border is about $\mathrm{T} 4$, the inferior scapular border is about $\mathrm{T} 7$, and the iliac crest is about the L4. The ROM of the affected side should also be compared with the unaffected side. It is important that the examiner stabilizes the scapula to evaluate true ROM of the glenohumeral joint. The scapulothoracic movement in relation to the glenohumeral joint movement during arm elevation is also important. The scapulohumeral rhythm is the ratio of the glenohumeral motion to the scapulothoracic motion. The ratio is commonly found to be $2: 1$, but scapula motion is reported to be highly variable in normal subjects and patients with symptomatic shoulder disease (20). Restoration and optimization of the scapulohumeral rhythm is a major goal in rehabilitation as it is important for shoulder function (21). ROM measurements can be affected by various body positions, such as sitting, standing, or supine positions (22). The sitting position may be useful for measuring a person's shoulder mobility during the performance of a functional task and to observe abnormal compensatory strategies. 
The supine position can be reliable as it controls for the confounding variable of pelvic position and eliminates the effects of gravity. Some physicians prefer to limit scapular movement to isolate glenohumeral movement while others do not, and the amount of motion measured may be different depending on whether the patient was sitting or lying supine (23). Patients with deltoid weakness may achieve greater ROM in the supine position than in the sitting position, as the force of gravity assists the motion after $90^{\circ}$. Similar intrarater reliability was reported when the shoulder ROM measurements were taken in the sitting or supine positions in a previous study by Sabari et al. (22). This study suggested that shoulder ROM must be measured in a consistent position and that the position of testing should be routinely recorded.

\section{Physical examination tests}

Physical examination tests for shoulder problems include muscular strength tests and provocative tests. Strength test is usually performed by comparing the shoulder muscles of the affected side and contralateral side. Physical examination tests of the shoulder are performed for diagnosing various shoulder diseases. Sensitivity, specificity, and likelihood ratios are used to provide data on the diagnostic accuracy of these tests. Sensitivity represents the proportion of actual positive results and specificity represents the proportion of negative results. Likelihood ratio (LR) is used to assess the clinical usefulness of a diagnostic test. The sensitivity and the specificity are combined in the LR into a ratio that quantifies the probability of the presence or absence of a disease in a negative or positive test. The positive LR+ is the probability of the condition being present when the test is positive whereas the negative likelihood ratios (LR-) is the probability of the condition being present when the test result is negative. An $L R>1$ indicates that the test result confirms the presence of a disease, whereas an $\mathrm{LR}<1$ indicates that the result confirms the absence of a disease (24). A large LR+ indicated that the condition is highly likely to be present, and a small LR- reflects that the condition is highly unlikely to be present. The diagnostic accuracy of physical examination tests is usually considered acceptable if LR $+\geq 2$ or LR- $\leq 0.50$ (25).

\section{Rotator cuff disorders}

Rotator cuff disease is a continuum of shoulder pathologies progressing from mild impingement to partial tear, fullthickness tear, massive tear, and, finally, arthropathy of the rotator cuff (26). Chronic impingement syndrome usually involves tears beginning on the bursal surface or within the tendon. Rotator cuff tears are prevalent, with asymptomatic cuff abnormalities reported in $30 \%$ of patients older than 60 years (27). The physical examination of patients with suspected rotator cuff disorders should include an evaluation of the cervical spine. Problems originating in the cervical spine are frequently misdiagnosed as symptoms of the shoulder and it is often clinically difficult to determine the exact source of pain (28). Assessment of both active and passive ROM is important as mentioned previously. Loss of active ROM with relative preservation of passive ROM is likely to suggest shoulder impingement or rotator cuff injury. Restriction of both passive and active ROM may indicate the presence of adhesive capsulitis, which may also be observed in impingement tests (25). Strength testing of the rotator cuff muscles should be performed to detect weakness that may result from a rotator cuff tear or tendinitis.

\section{Subacromial impingement test}

Subacromial impingement refers to the rotator cuff tendons and bursa being pinched between the greater tuberosity of the humerus, acromion, and coracoacromial ligament with arm elevation (29). First, in the painful arc test, the patient is instructed to elevate the arm in the scapular plane. Provocation of pain between $70^{\circ}$ and $120^{\circ}$ during elevation yields a positive test (25). Second, the Neer's impingement test is performed with the examiner using one hand to fix the scapula, while with the other hand, the patient's arm is elevated and internally rotated (29) (Figure 1A). The pain is provoked as the greater tubercle contacts the roof of the shoulder joint and the volume of the subacromial space is decreased. The test result is positive if pain is present around the anterior shoulder. Third, the Hawkins-Kennedy impingement test is performed with the patient's arm and elbow flexed to $90^{\circ}(29)$ (Figure 1B). The test result is positive when there is pain around the anterior or lateral shoulder as the examiner internally rotates the arm. This test narrows the subacromial space between the greater tubercle and the coracoacromial ligament. These two tests have shown high sensitivity rather than specificity $(29,30)$ and are recommended for ruling out subacromial impingement when negative (Table 1). 

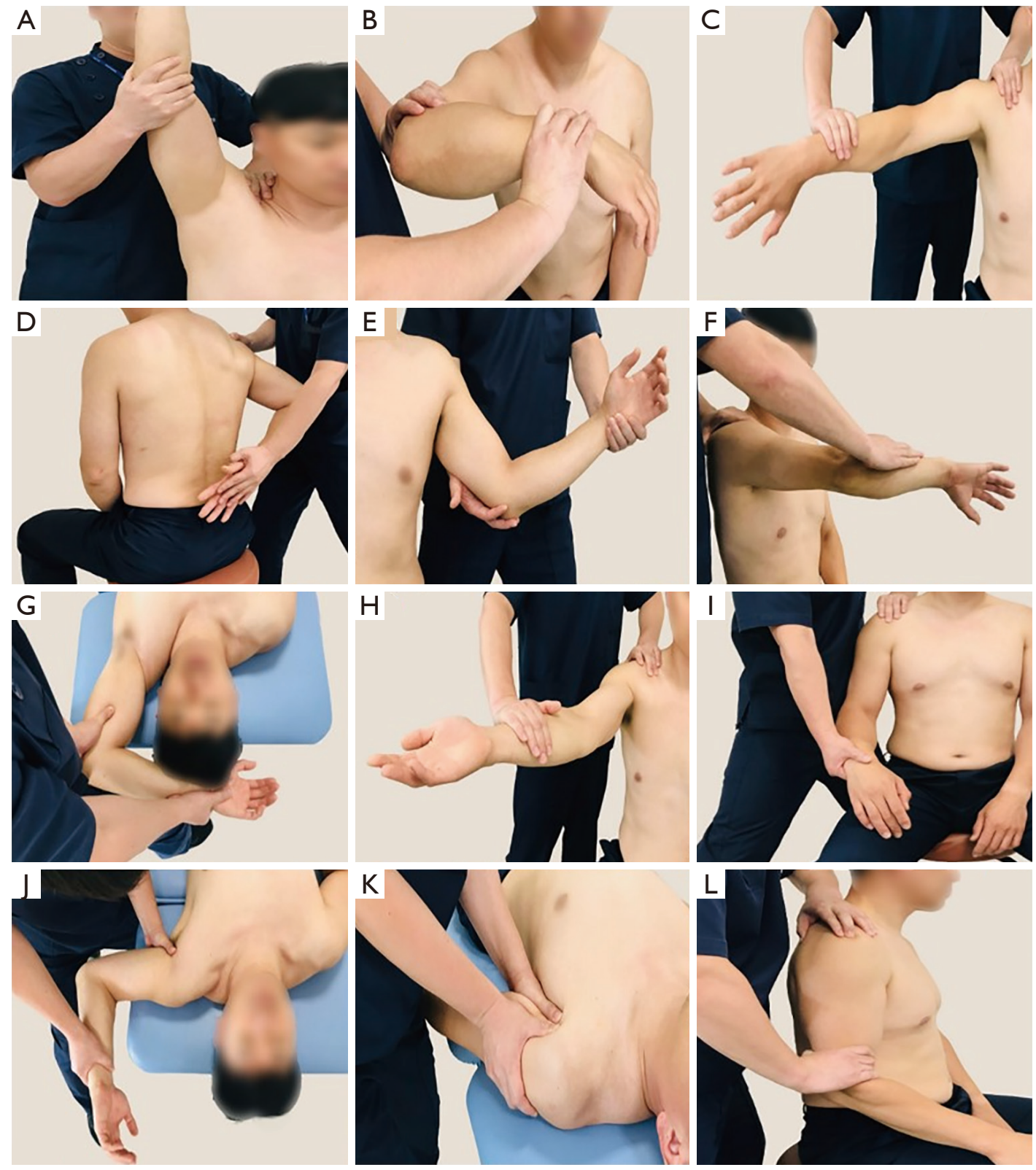

Figure 1 (A) Neer impingement test. (B) Hawkins-Kennedy impingement test. (C) Empty can test. (D) Lift-off test. (E) Resisted external rotation lag sign. (F) O'Brien test. (G) Crank test. (H) Speed test. (I) Yergason test. (J) Apprehension test. (K) Anterior and posterior drawer test. (L) Sulcus sign.

\section{Tendon integrity test}

\section{Supraspinatus}

The evaluation of the supraspinatus muscle, which is commonly affected in rotator cuff injury, is performed with the empty can test, also known as Jobe's test. The arm is abducted to $90^{\circ}$ and the shoulder is internally rotated with the forearm maximally pronated, making the thumb point down (Figure 1C). A positive result is pain or weakness of the arm as the examiner applies downward force to the arm. The test showed high sensitivity and specificity with LR+ of more than 2 and LR- of less than $0.5(1,25,39)$ and is recommended as a screening and confirmatory test for large or massive full-thickness tear (Table 1). The test is repeated with the arm in external rotation, making the thumb point 
Table 1 Diagnostic value of physical examination tests for shoulder

\begin{tabular}{|c|c|c|c|c|}
\hline Name of special test & Sensitivity (\%) & Specificity (\%) & LR+ & LR- \\
\hline \multicolumn{5}{|l|}{ Impingement tests } \\
\hline Neer impingement test (30) & 72 & 60 & 1.79 & 0.47 \\
\hline Hawkin's test (30) & 80 & 56 & 1.84 & 0.35 \\
\hline Painful arc (30) & 53 & 76 & 2.25 & 0.62 \\
\hline Empty can test (31) & 88 & 70 & 2.93 & 0.17 \\
\hline Lift-off test (32) & 18 & 92 & 2.3 & 0.89 \\
\hline Belly-press test (32) & 25 & 97.9 & 11.90 & 0.77 \\
\hline Belly-off test (30) & 86 & 91 & 9.67 & 0.14 \\
\hline \multicolumn{5}{|l|}{ SLAP tests } \\
\hline O’Brien test (30) & 67 & 37 & 1.06 & 0.89 \\
\hline Crank test (30) & 34 & 75 & 1.36 & 0.88 \\
\hline \multicolumn{5}{|l|}{ Biceps tendon tests } \\
\hline Speed test (35) & 54 & 81 & 2.77 & 0.58 \\
\hline Yergason's test (35) & 41 & 79 & 1.94 & 0.74 \\
\hline Upper cut test (35) & 73 & 78 & 3.38 & 0.34 \\
\hline \multicolumn{5}{|l|}{$A C$ joint tests } \\
\hline Anterior drawer test $(37,38)$ & $53-58.3$ & $85-92.7$ & $3.57-7.95$ & $0.45-0.56$ \\
\hline
\end{tabular}

LR+, positive likelihood ratios; LR-, negative likelihood ratios; AC, acromioclavicular, SLAP, superior labrum anterior to posterior.

upwards. This is known as the full can test.

\section{Subscapularis}

Four tests are commonly used to evaluate the subscapularis. First, the lift-off test, also known as the Gerber test (Figure 1D), is performed. The arm is internally rotated with the elbow flexed and the hand is placed on the lumbar spine. A positive result is the inability to lift the dorsum of the hand off the back without using the triceps muscle. Second, the belly-press test, which is also called the Napoleon sign, can be performed, which involves resting the hand on the abdomen with arm internally rotated and elbow flexed with the wrist in neutral position. A positive result is the inability to maintain the elbow forward and the wrist neutral when the patient is asked to press the abdomen. Third, the bellyoff test is performed with the elbow in flexion to $90^{\circ}$ with the palm over the belly. The examiner moves the elbow forward, placing the arm into maximum internal rotation of the shoulder. A positive result is inability to keep the wrist straight and maintain the position of internal rotation as the examiner releases the wrist. Fourth, the bear hug test is performed by asking patient to grasp the contralateral shoulder with the affected arm in forward flexion and 
elbow flexion across the anterior chest. A positive result is inability to maintain a downward force as the examiner applies resisted pull-off. The performances of the affected and healthy sides are compared. The lift-off and the bellypress tests showed high specificities and LR+, and thus recommended as confirmatory tests $(32,40)$. The belly-off test showed high LR+ (9.67) and low LR- (0.14), supporting the diagnosis of a subscapularis tear $(30,41)$.

\section{Infraspinatus and teres minor}

The evaluation of the infraspinatus muscle and teres minor is performed with resisted external rotation. The external rotation lag sign involves the arm positioned in $20^{\circ}$ of flexion and the elbow flexed to $90^{\circ}$ (Figure 1 E). A positive lag sign is marked when the examiner externally rotates the arm to its maximum extent and patient is unable to maintain the arm in this position. This test showed high sensitivity and LR+ and low LR- and is recommended as a screening test $(25,31,39)$. It was reported to be useful in detecting a combined full-thickness tear of the supraspinatus and infraspinatus with high LR+ (13.36) and low LR- (0.03) (33). The Hornblower's test, also known as the Patte test, is also performed. The arm is passively abducted with elbow flexion to $90^{\circ}$ with the examiner's support. The patient is asked to rotate the arm externally. A positive result is the inability to maintain the position. The Hornblower's test was recommended as a screening and confirmatory test, as it showed high sensitivity, specificity, and LR+ and low LR$(34,40)$. Both the infraspinatus and teres minor muscle help stabilize the glenohumeral joint and contribute to external rotation of the humerus. It is hard to clearly distinguish the teres minor from the infraspinatus, as they both share similar function. Therefore, it is often difficult to identify the weakness of the isolated infraspinatus or teres minor based solely on physical examination (14).

\section{Superior labrum anterior to posterior lesions}

In superior labrum anterior to posterior (SLAP) lesions, the labral tear extends anteriorly from the posterior superior labrum and the superior labrum detaches along with the biceps tendon (42). SLAP lesions are often assessed using the O'Brien test. The patient's arm is flexed to $90^{\circ}$ and slightly adducted with the forearm pronated and internally rotated, making the thumb pointing down (Figure $1 F$ ). A positive result is pain or weakness when the examiner applies downward force on the arm. The test is continued by externally rotating the arm with forearm supination, making the thumb point upwards.
Again, the examiner applies downward force on the arm. If pain is reduced in this position, labral pathology may be suspected. The Crank test is performed in suspected labral tears (Figure 1G). The patient lies supine with the arm elevated to $160^{\circ}$ in the scapular plane. An axial load is applied to the glenohumeral joint as the humerus is internally and externally rotated. A positive result is pain with or without a click. Both tests are commonly used as examination tests for SLAP tears but both tests showed insufficient evidence to accurately predict SLAP lesions with moderate sensitivities and specificities across previous studies $(36,40,43)$.

\section{Lesions in the biceps tendon}

Lesions in the long head of the biceps tendon can cause pain in the anterior shoulder. The evaluation of the long head of the biceps tendon begins with palpation. The speed test is performed with the patient's shoulder flexed, elbow extended, and forearm supinated (Figure 1H). The examiner applies a downward force to the arm. If pain is present along the biceps tendon or within the bicipital groove, the test result is considered positive. The Yergason test is also used to identify lesions in the long head of biceps tendon. This test is performed with the patient's arm adducted in neutral rotation and elbow flexed to $90^{\circ}$ (Figure 1I). The test result is considered positive if pain is present in the biceps tendon or bicipital groove with resisted supination, with high LR+ (3.0) (44). Both the speed test and Yergason test are being increasingly used in diagnosing unstable SLAP lesions (30). Additionally, the upper cut test is performed to detect lesions in the biceps tendon by having the patient make a fist with the elbow flexed $90^{\circ}$ with forearm supination. The examiner resists against the patient's fist with his or her hand as the patient brings his or her hand up and toward the examiner's chin. In a previous study by Ben Kibler et al. (35), the upper cut and speed test reported LR+ of 3.38 and 2.77, respectively, and the upper cut, Yergason, and speed tests are reported to have high specificities.

\section{Acromioclavicular joint disease}

The evaluation of the acromioclavicular AC joint begins with palpating over the AC joint. The AC joint is a gliding or plane-style synovial joint, formed at the junction of the clavicle and acromion. A well-known test for the evaluation of AC joint is the O'Brien test, which has been mentioned previously. In SLAP lesions, the pain is felt deep inside 
the shoulder, whereas in AC joint disorder, pain is felt on top of the shoulder, on testing with the thumb pointing downwards. The AC joint is also evaluated with the crossbody adduction test. The arm and elbow are extended across the chest by the examiner. A positive test result is anterior shoulder pain or pain around the AC joint. Tenderness on palpation of the AC joint, which seems to be the easiest and the most effective method, was recommended as a screening test for AC joint disease as it showed high sensitivity (96\%) and low LR- $(0.4)(31,45)$.

\section{Instability}

Glenohumeral instability frequently occurs in young adults. The glenohumeral joint is very mobile and has a wide ROM. To protect the joint, the shoulder has various static and dynamic stabilizers, which provide stability in various positions (36). Instability is a pathologic process that involves a symptomatic increase in humeral head translation relative to the glenoid. This leads to pain, impairment of physical function, and weakness (1). When shoulder instability is suspected, the examiner should also look for evidence of excessive laxity and translation. The Beighton score is used to quantify joint laxity and hypermobility. It is a nine-point scale and its diagnostic thresholds vary from 4 to 6 or above (46). It is important to address the presence of general hyperlaxity because shoulder dislocation may manifest as the first symptom of joint hypermobility syndrome, such as EhlersDanlos or Marfan's syndrome (47). Physical examination tests for instability should always include comparison with the contralateral shoulder.

\section{Anterior/posterior instability}

The evaluation of anterior instability is performed with the apprehension test. The patient stands or lies supine with the arm in abduction and elbow flexion to $90^{\circ}$. The arm is placed off of the bed as the examiner applies external rotation force, stabilizing the scapula (Figure 17). The evaluation of posterior instability is performed with the shoulder flexed forward to $90^{\circ}$ maximally and internally rotated as the examiner applies a posteriorly directed force on the patient's elbow. A positive test is patient's report of apprehension or pain. The Jobe relocation test can be performed in a similar manner, placing a posterior force on the humeral head. A positive test is feeling relief of apprehension or pain. The load and shift test is also performed to detect anterior-posterior instability of the glenohumeral joint. With the patient seated with the hand resting on the thigh, the examiner' stabilizes the scapula with one hand and uses the other hand to push the humeral head into the glenoid, generating the "load". The examiner shifts the humeral head anteriorly and posteriorly. A positive result is excessive translation or feeling of apprehension. These tests showed high sensitivity (71.7\% to $98.3 \%)$, specificity ( $71.6 \%$ to $96 \%$ ), and LR+ (3.46 to 20.22 ) and low LR- $(<0.5)$, and were useful for diagnosing shoulder instability (36-38).

Another test performed to assess the amount of humeral head translation is the anterior and posterior drawer test. The patient lies supine, with the shoulder in $80-120^{\circ}$ abduction, $0-20^{\circ}$ flexion, and $0-30^{\circ}$ external rotation. The examiner places one hand in the patient's axilla with the fingers around the humerus and the other hand is placed over the lateral aspect of the upper arm (Figure $1 \mathrm{~K}$ ). Then the examiner pulls the humerus anteromedially (anterior glide) or posterolaterally (posterior glide). A positive test is pain, apprehension, and/or increased ROM. This test was recommended as a confirmatory test with high specificity and $\mathrm{LR}+(37,38)$.

\section{Inferior instability}

The sulcus sign is evaluated to detect the presence of inferior instability of the glenohumeral joint. The examiner applies a downward force at the elbow on the patient's relaxed upper arm (Figure $1 L$ ). A positive test is a sulcus or a depression visible between the lateral edge of the acromion and the head of the humerus. Although commonly used in clinical practice, the data on its efficacy for diagnosing inferior instability is unavailable (36).

\section{Combination of tests}

In some cases, isolated tests may be insufficient to achieve an accurate diagnosis. In such cases, the combination of special tests may improve the diagnostic accuracy of physical examination of the shoulder. For example, Michener et al. suggested that $\geq 3$ positive results in the painful arc, Neer impingement, Hawkins-Kennedy impingement, external rotation resistance, or empty can test can confirm the diagnosis of subacromial impingement syndrome with high LR+ (2.93) and low LR- values $(0.34)(30,48)$. Chew et al. suggested that a diagnosis of supraspinatus pathology may be accomplished with a cluster of three tests as follows: age $>39$ years, positive sign of painful arc, and self-reported clicking or popping with a high LR+ value (32.20) (49). Similarly, Litaker et al. also suggested that the presence 
of the following 3 signs may suggest the presence of a rotator cuff tear: age $\geq 65$ years, night pain, and weakness on external rotation with a high $\mathrm{LR}+$ value (9.84) (50). A combination of the upper cut and speed tests was reported to be beneficial for detecting lesions in the biceps (36). The combination of these specific tests may be used to provide better accuracy along with comprehensive clinical examinations, including history and symptoms.

\section{Imaging tests}

Imaging modalities, including radiography, ultrasound (US), and magnetic resonance imaging (MRI), are often used for the evaluation of shoulder pathologies and facilitation of better diagnosis (51). Radiography provides important information regarding osseous structures and secondary signs of soft tissue pathology around the shoulder joint. In combination with symptoms and physical examination tests, radiographs can guide further imaging such as US and MRI. US enables visualization of soft tissues (e.g., tendons and muscles) and detection of fluids (e.g., in effusion and bursitis). The real-time capability of US also enables USguided injections. MRI is superior to US in visualizing osseous structures, the bone marrow, and the overlying cartilage (51). Both US and MRI are reported as highly accurate in the diagnosis of rotator tears $(51,52)$. Although these modalities are considered as the gold standard for the evaluation of shoulder problems, they are frequently overused (53). It is also important for physicians to consider the costs of ordering imaging tests because a higher diagnostic accuracy, though beneficial, is not always needed. Unnecessary tests can be avoided by utilizing patients' history and physical examination tests in approaching the underlying mechanisms of shoulder problems, which could mean substantial savings for an already stretched health care system (53).

This review explored the diagnostic accuracy of special tests previously reported in the literature. Advanced imaging tests are highly beneficial, yet physicians must exercise prudence when ordering them. Imaging tests should be performed only when there is an additional need to confirm or reject suspected diagnoses derived from patients' history and physical examination tests. Even so, physicians must realize that many studies describing physical examination tests are rather insufficient to make accurate diagnosis. There are limitations to physical examination tests because these tests are often operator-dependent and are dependent on a subjective outcome, such as a patient's report of pain (29). In addition, physical examination tests may be nonspecific, as similar results can be produced by multiple shoulder entities (29). However, considering that imaging tests are time-consuming and expensive as previously mentioned, a combination of history and physical examination should be first considered before imaging tests are ordered. Further studies directly comparing the diagnostic accuracy of both physical examination tests and imaging tests are needed in the future.

\section{Conclusions}

Shoulder pain is a common musculoskeletal problem that causes significant pain and disability. The evaluation of shoulder pain can be very complex, but it seems unnecessary and time consuming to perform every physical examination technique for every patient. The clinician should rather focus on choosing the best test, based on the patient's history, to confirm a suspected diagnosis. These tests in isolation are insufficient to achieve an accurate diagnosis. The combination of the special tests may improve the diagnostic accuracy of the physical examination of the shoulder. Patient history and diagnostic imaging should be considered to improve the accuracy if necessary.

\section{Acknowledgments}

Funding: The present study was supported by a National Research Foundation of Korea grant funded by the Korean government (grant No. NRF-2019M3E5D1A02069399).

\section{Footnote}

Reporting Checklist: The authors have completed the Narrative Review reporting checklist. Available at http:// dx.doi.org/10.21037/apm-20-1808

Conflicts of Interest: All authors have completed the ICMJE uniform disclosure form (available at http://dx.doi. org/10.21037/apm-20-1808). The authors have no conflicts of interest to declare.

Ethical Statement: The authors are accountable for all aspects of the work in ensuring that questions related to the accuracy or integrity of any part of the work are 
appropriately investigated and resolved.

Open Access Statement: This is an Open Access article distributed in accordance with the Creative Commons Attribution-NonCommercial-NoDerivs 4.0 International License (CC BY-NC-ND 4.0), which permits the noncommercial replication and distribution of the article with the strict proviso that no changes or edits are made and the original work is properly cited (including links to both the formal publication through the relevant DOI and the license). See: https://creativecommons.org/licenses/by-nc-nd/4.0/.

\section{References}

1. Bakhsh W, Nicandri G. Anatomy and Physical Examination of the Shoulder. Sports Med Arthrosc Rev 2018;26:e10-e22.

2. Lange T, Matthijs O, Jain NB, et al. Reliability of specific physical examination tests for the diagnosis of shoulder pathologies: a systematic review and meta-analysis. Br J Sports Med 2017;51:511-8.

3. Halder AM, Kuhl SG, Zobitz ME, et al. Effects of the glenoid labrum and glenohumeral abduction on stability of the shoulder joint through concavity-compression : an in vitro study. The Journal of bone and joint surgery. J Bone Joint Surg Am 2001;83:1062-9.

4. Familiari F, Huri G, Simonetta R, et al. SLAP lesions: current controversies. EFORT Open Rev 2019;4:25-32.

5. Cook TS, Stein JM, Simonson S, et al. Normal and variant anatomy of the shoulder on MRI. Magn Reson Imaging Clin N Am 2011;19:581-94.

6. Michener LA, McClure PW, Karduna AR. Anatomical and biomechanical mechanisms of subacromial impingement syndrome. Clin Biomech (Bristol, Avon) 2003;18:369-79.

7. Bishay V, Gallo RA. The evaluation and treatment of rotator cuff pathology. Prim Care 2013;40:889-910.

8. Ko JY, Wang FS. Rotator cuff lesions with shoulder stiffness: updated pathomechanisms and management. Chang Gung Med J 2011;34:331-40.

9. Harrison AK, Flatow EL. Subacromial impingement syndrome. J Am Acad Orthop Surg 2011;19:701-8.

10. Bigliani LU, Dalsey RM, McCann PD, et al. An anatomical study of the suprascapular nerve. Arthroscopy 1990;6:301-5.

11. Kostretzis L, Theodoroudis I, Boutsiadis A, et al. Suprascapular Nerve Pathology: A Review of the Literature. Open Orthop J 2017;11:140-53.

12. Cothran RL Jr, Helms, C. Quadrilateral space syndrome: incidence of imaging findings in a population referred for MRI of the shoulder. AJR Am J Roentgenol 2005;184:989-92.

13. Hangge PT, Breen I, Albadawi H, et al. Quadrilateral Space Syndrome: Diagnosis and Clinical Management. J Clin Med 2018;7:86.

14.Wilson L, Sundaram M, Piraino DW, et al. Isolated teres minor atrophy: manifestation of quadrilateral space syndrome or traction injury to the axillary nerve? Orthopedics 2006;29:447-50.

15. Friend J, Francis S, McCulloch J, et al. Teres minor innervation in the context of isolated muscle atrophy. Surg Radiol Anat 2010;32:243-9.

16. House J, Mooradian A. Evaluation and management of shoulder pain in primary care clinics. South Med J 2010;103:1129-35; quiz 1136-7.

17. Burbank KM, Stevenson JH, Czarnecki GR, et al. Chronic shoulder pain: part I. Evaluation and diagnosis. Am Fam Physician 2008;77:453-60.

18. Ferri M, Finlay K, Popowich T, et al. Sonography of full-thickness supraspinatus tears: comparison of patient positioning technique with surgical correlation. AJR. AJR Am J Roentgenol 2005;184:180-4.

19. Jacobson JA. Shoulder US: anatomy, technique, and scanning pitfalls. Radiology 2011;260:6-16.

20. Flores-Hernandez C, Eskinazi I, Hoenecke HR, et al. Scapulothoracic rhythm affects glenohumeral joint force. JSES Open Access 2019;3:77-82.

21. van der Helm FC. Analysis of the kinematic and dynamic behavior of the shoulder mechanism. J Biomech 1994;27:527-50.

22. Sabari JS, Maltzev I, Lubarsky D, et al. Goniometric assessment of shoulder range of motion: comparison of testing in supine and sitting positions. Arch Phys Med Rehabil 1998;79:647-51.

23. Lee SH, Yoon C, Chung SG, et al. Measurement of Shoulder Range of Motion in Patients with Adhesive Capsulitis Using a Kinect. PLoS One 2015;10:e0129398.

24. McGee S. Simplifying likelihood ratios. J Gen Intern Med 2002;17:646-9.

25. Hippensteel KJ, Brophy R, Smith MV, et al. A Comprehensive Review of Physical Examination Tests of the Cervical Spine, Scapula, and Rotator Cuff. J Am Acad Orthop Surg 2019;27:385-94.

26. Sambandam SN, Khanna V, Gul A, et al. Rotator cuff tears: An evidence based approach. World J Orthop 2015;6:902-18.

27. Dang A, Davies M. Rotator Cuff Disease: Treatment 
Options and Considerations. Sports Med Arthrosc Rev 2018;26:129-33.

28. Bokshan SL, DePasse JM, Eltorai AE, et al. An EvidenceBased Approach to Differentiating the Cause of Shoulder and Cervical Spine Pain. Am J Med 2016;129:913-8.

29. O'Kane JW, Toresdahl BG. The evidenced-based shoulder evaluation. Curr Sports Med Rep 2014;13:307-13.

30. Hegedus EJ, Goode AP, Cook CE, et al. Which physical examination tests provide clinicians with the most value when examining the shoulder? Update of a systematic review with meta-analysis of individual tests. Br J Sports Med 2012;46:964-78.

31. Hughes PC, Taylor NF, Green RA. Most clinical tests cannot accurately diagnose rotator cuff pathology: a systematic review. Aust J Physiother 2008;54:159-70.

32. Barth JR, Burkhart SS, De Beer JF. The bear-hug test: a new and sensitive test for diagnosing a subscapularis tear. Arthroscopy 2006;22:1076-84.

33. Castoldi F, Blonna D, Hertel R. External rotation lag sign revisited: accuracy for diagnosis of full thickness supraspinatus tear. J Shoulder Elbow Surg 2009;18:529-34.

34. Collin P, Treseder T, Denard PJ, et al. What is the Best Clinical Test for Assessment of the Teres Minor in Massive Rotator Cuff Tears? Clin Orthop Relat Res 2015;473:2959-66.

35. Ben Kibler W, Sciascia AD, Hester $\mathrm{P}$, et al. Clinical utility of traditional and new tests in the diagnosis of biceps tendon injuries and superior labrum anterior and posterior lesions in the shoulder. Am J Sports Med 2009;37:1840-7.

36. Hippensteel KJ, Brophy R, Smith MV, et al. Comprehensive Review of Provocative and Instability Physical Examination Tests of the Shoulder. J Am Acad Orthop Surg 2019;27:395-404.

37. Farber AJ, Castillo R, Clough M, et al. Clinical assessment of three common tests for traumatic anterior shoulder instability. J Bone Joint Surg Am 2006;88:1467-74.

38. van Kampen DA, van den Berg T, van der Woude HJ, et al. Diagnostic value of patient characteristics, history, and six clinical tests for traumatic anterior shoulder instability. J Shoulder Elbow Surg 2013;22:1310-9.

39. Hertel R, Ballmer FT, Lombert SM, et al. Lag signs in the diagnosis of rotator cuff rupture. J Shoulder Elbow Surg 1996;5:307-13.

40. Hegedus EJ, Goode A, Campbell S, et al. Physical examination tests of the shoulder: a systematic review with meta-analysis of individual tests. Br J Sports Med 2008;42:80-92; discussion 92.

41. Bartsch M, Greiner S, Haas NP, et al. Diagnostic values of clinical tests for subscapularis lesions. Knee Surg Sports Traumatol Arthrosc 2010;18:1712-7.

42. Sarkkaya B, Bozkurt C, Gül O, et al. Comparison of the clinical results of isolated Bankart and SLAP 5 lesions after arthroscopic repair. Jt Dis Relat Surg 2020;31:223-9.

43. Parentis MA, Glousman RE, Mohr KS, et al. An evaluation of the provocative tests for superior labral anterior posterior lesions. Am J Sports Med 2006;34:265-8.

44. Guanche CA, Jones DC. Clinical testing for tears of the glenoid labrum. Arthroscopy 2003;19:517-23.

45. Walton J, Mahajan S, Paxinos A, et al. Diagnostic values of tests for acromioclavicular joint pain. J Bone Joint Surg Am 2004;86:807-12.

46. Juul-Kristensen B, Røgind H, Jensen DV, et al. Interexaminer reproducibility of tests and criteria for generalized joint hypermobility and benign joint hypermobility syndrome. Rheumatology (Oxford, England) 2007;46:1835-41.

47. Nourissat G, Vigan M, Hamonet C, et al. Diagnosis of Ehlers-Danlos syndrome after a first shoulder dislocation. J Shoulder Elbow Surg 2018;27:65-9.

48. Michener LA, Walsworth MK, Doukas WC, et al. Reliability and diagnostic accuracy of 5 physical examination tests and combination of tests for subacromial impingement. Arch Phys Med Rehabil 2009;90:1898-1903.

49. Chew K, Pua Y, Chin J, et al. Clinical predictors for the diagnosis of supraspinatus pathology. Physiotherapy Singapore 2010;13:12-7.

50. Litaker D, Pioro M, El Bilbeisi H, et al. Returning to the bedside: using the history and physical examination to identify rotator cuff tears. J Am Geriatr Soc 2000;48:1633-7.

51. Gimarc DC, Lee KS. Shoulder MR Imaging Versus Ultrasound: How to Choose. Magn Reson Imaging Clin N Am 2020;28:317-30.

52. Jacobson JA. Musculoskeletal ultrasound: focused impact on MRI. AJR Am J Roentgenol 2009;193:619-27.

53. Sheehan SE, Coburn JA, Singh H, et al. Reducing Unnecessary Shoulder MRI Examinations Within a Capitated Health Care System: A Potential Role for Shoulder Ultrasound. J Am Coll Radiol 2016;13:780-7.

Cite this article as: Yang S, Kim TU, Kim DH, Chang MC. Understanding the physical examination of the shoulder: a narrative review. Ann Palliat Med 2021;10(2):2293-2303. doi: 10.21037/apm-20-1808 